\title{
Racionalismo y certeza moral en Descartes*
}

\section{Rationalism and moral certainty in Descartes}

\author{
Salvador Jara Guerrero ${ }^{\dagger}$
}

\begin{abstract}
Resumen
Clasificar las corrientes de pensamiento en un esquema binario se ha convertido en un hábito que reduce la complejidad de las ideas a dos extremos y con ello simplifica también las ideas de los pensadores. Esta tendencia ha clasificado a René Descartes como el padre del racionalismo. Sin embargo, una revisión más cuidadosa de su obra muestra otra faceta menos rígida, más prudente y un interés especial por el trabajo interdisciplinario. Esta actitud prudencial que Descartes nos muestra en el Tratado de la luz concuerda con la preocupación por la prudencia y la tolerancia de algunos pensadores de la época. Pero el interés en este trabajo es el de desmitificar la figura dogmática que con frecuencia se achaca a Descartes.
\end{abstract}

Palabras clave: Descartes - certidumbre moral - racionalismo - ciencia - filosofía

\begin{abstract}
It has been a habit to classify different schools of thought in a binary scheme, reducing the complexity of the ideas to two ends and also reducing the philosopher's thought to one of the poles. This practice has classified René Descartes as the father of rationalism. Nevertheless, a more careful review of his work shows another face, less rigid, more prudent and a special interest for interdisciplinary work. Descartes' prudential attitude is shown in his Treatise of the Light. Other philosophers shared with him this attitude and special concern for prudence and tolerance at that time. The aim of this work is to demystify the dogmatic rationalism that has frequently been attributed to Descartes.
\end{abstract}

Keywords: Descartes - moral certainty - rationalism - science - philosophy

\footnotetext{
* Recibido: 9 de Enero de 2012. Aceptado: 25 de Febrero de 2012.

† Facultad de Ciencias Físico Matemáticas/Instituto de Investigaciones Filosóficas, Universidad Michoacán de San Nicolás de Hidalgo. Para contactar al autor, por favor escriba a: sjarag@gmail.com. Metatheoria 2(2) (2012): 81-90. ISSN 1853-2322.

(c) Editorial de la Universidad Nacional de Tres de Febrero. Publicado en la República Argentina.
} 
La historia de la filosofía o de los problemas filosóficos siempre será contemporánea, porque solo un minúsculo porcentaje de la nueva filosofía sobrevivirá quizá un par de generaciones, en cambio, los problemas clásicos nos han mostrado que resisten el paso del tiempo en un porcentaje muy alto.

Algunos de esos problemas tradicionales se refieren a la naturaleza del conocimiento, ise trata de un descubrimiento de algo ya dado y existente con independencia del sujeto o es un invento, una construcción en la que buscamos una correspondencia de nuestras creencias con la realidad?

Íntimamente vinculada con lo anterior está la cuestión acerca de los criterios o parámetros (si es que los hay) que sustentan nuestras creencias, las maneras en que confirmamos o justificamos nuestras pretensiones de conocimiento. ¿Existen conocimientos indudables que nos puedan servir de base y fundamento para la construcción de los demás? Y si los hay, ¿son conocimientos derivados de la experiencia o de la razón? Y si no existieran tales fundamentos, ¿son los conocimientos como nudos en una red que se dan sustento mutuamente, siendo la coherencia del sistema su mejor justificación? Y por otra parte, están los problemas acerca de la existencia y el conocimiento de entidades no observables. No se trata, por supuesto, de problemas simples ni pequeños, al contrario, sus ramificaciones e implicaciones son muchas y muy profundas.

René Descartes es conocido fundamentalmente por su racionalismo y su apego a principios que expliquen los fenómenos observados. Ha sido reconocido por la fundamentación metafísica de sus principios. Sin embargo, poco se ha dicho acerca del estatuto de certeza de sus escritos físicos y en la explicación de los fenómenos particulares; mientras que los principios físicos son absolutamente verdaderos, las hipótesis físicas poseen solamente certeza relativa (Turró 1989). El objetivo de este trabajo es destacar esta certeza problemática en una de sus obras: El mundo. Tratado de la luz.

Es importante observar en primer lugar la analogía entre los argumentos utilizados en el derecho y los usados en filosofía natural durante la Revolución Científica. La "ley común" inglesa, usada para los juicios de asesinato y traición, no consistía en un sistema simple y susceptible de codificación sino, en su mayoría, en una gran colección de casos anteriores. Es decir, se trataba de una ley basada fundamentalmente en la jurisprudencia. Por tanto, la certeza estaba fundamentada en la experiencia acumulada (Sargent 1995, pp. 42-61). Pero ya no se trataba solo de la experiencia de un individuo, sino de un grupo heterogéneo de personas: el jurado, donde el objetivo era buscar la concurrencia de probabilidades para alcanzar la certeza moral.

Mientras que la ley romana requería de la prueba total, probatio plena, que solo se satisfacía con la confesión, así fuera con tortura, la ley común requería un juicio público con jurados. Estos con frecuencia se enfrentaban a evidencia poco familiar y casi siempre circunstancial, por lo que cada vez más debían considerar los testimonios de los testigos paralelamente con su calidad moral. Consecuentemente, los jurados debían evaluar, con la evidencia, la credibilidad de ambos, testigo y testimonio (Shapiro 1991, p. 6). En el primer caso, con la confe- 
sión, el jurado se convencía de la culpabilidad plena del acusado. En el segundo, sin importar si se le declaraba culpable o inocente, el fallo siempre sería una conjetura, una inducción a partir de la evidencia; aunque nunca se tendría la certeza de una demostración, siempre se eliminaría la duda de manera razonable.

Estas prácticas y teorías legales del siglo XVII relacionadas con la credibilidad del testimonio y el diseño de los tribunales se encuentran relacionadas con las primeras expresiones de la probabilidad matemática, de las que tomaron, de acuerdo con Daston, dos características: la interpretación epistémica de la probabilidad como grado de certidumbre y la primacía del concepto de esperanza o expectativa (Daston 1988, p. 6). La probabilidad constituía una herramienta útil que servía de guía para la acción, sobre todo respecto de lo relacionado con los préstamos, los juegos y las inversiones relativas a las importaciones, cuyo precio fluctuaba con frecuencia. Nuevamente con esto se destaca el importante papel de lo cotidiano y de la acción práctica; la experiencia toma el papel de evidencia o prueba que puede ser usada como un fundamento o base, para después ser retroalimentada y reinterpretada a la luz de las nuevas experiencias.

Así, los criterios de "más allá de la duda razonable" y de la "certeza moral", tan arraigados hoy en las cortes anglo-americanas, parecen haber sido producto del esfuerzo realizado, desde el siglo XVII, por encontrar criterios de evidencia y prueba en varias áreas del conocimiento, desde la religión hasta la filosofía natural, como un punto medio entre certeza y opinión.

Afirma Barbara J. Shapiro que, en general, se reconocían tres tipos de certidumbre o conocimiento, durante el siglo XVII (Shapiro 1991, p. 8):

a) Matemático, establecido o derivado de demostraciones lógicas o pruebas geométricas.

b) Físico, derivado de los datos sensoriales o de principios físicos.

c) Y moral, que se basaba tanto en testimonios como en reportes de datos sensoriales, se alcanzaba como una concurrencia de probabilidades en el sentido de evidencia convergente. Esta certidumbre moral, en la que asentían aquellos "cuyo juicio estaba libre de prejuicios", o se consideraba como indudable para cualquier persona razonable, era la más relevante para el derecho, la historia y la filosofía natural.

Especialmente este último criterio muestra una flexibilidad y prudencia que se aleja de los extremos dogmáticos que hoy día identificamos con el cientificismo.

Sin embargo, en el análisis posterior de la historia de los conocimientos es común encontrar la construcción de esquemas binarios en los que se presentan las ideas en blanco y negro, como polos opuestos. Así, podemos oponer el descubrimiento a la invención, el fundamentalismo unívoco y objetivo a un coherentismo subjetivo, el empirismo al racionalismo, las ciencias naturales a las humanidades, el método científico a la hermenéutica, el destino al libre albedrío, las leyes deterministas universales de la física al azar, lo global a lo local, el universalismo al relativismo, la separación y el control de variables al holismo o la naturaleza a la cultura. Y de igual forma es también común reducir las ideas de los pensadores a dos polos. 
La interesante polémica entre Robert Boyle y Thomas Hobbes durante el siglo XVII ilustra el caso. En su debate en torno al objetivo de la filosofía experimental es posible ubicarlos en dos extremos. Sin embargo, en otros ámbitos ninguno de los dos sostiene el mismo punto de vista (Jara 2009). Mientras que Hobbes mantenía que la filosofía experimental nunca llevaría al grado de certidumbre requerido por la filosofía natural, Boyle, en cambio, consideraba que el mundo es demasiado complicado para lograr conocerlo con absoluta certeza, pero estaba también convencido de que con el tiempo el conocimiento se acercaría cada vez más a las verdaderas causas de los efectos naturales (Boyle 1996, p. 15). Esa complejidad del mundo hizo pensar a Boyle que no era posible explicarlo por medio de las matemáticas; sin embargo, reconocía su utilidad para describir fenómenos, pero afirmaba que las matemáticas no nos dan las razones por las que los cuerpos actúan de la manera en que lo hacen; por tanto, un tratamiento matemático de los fenómenos naturales nos daría como resultado un sistema, dando lugar a reglas matemáticas y no a explicaciones causales (Hooykaas 1949, p. 40 y Sargent 1995, p. 66). Para Boyle el objetivo de la filosofía no era encontrar la certidumbre absoluta que Hobbes pretendía, sino la búsqueda de explicaciones inteligibles acerca de los mecanismos que operan debajo de los fenómenos (Boyle 1991, p. 128).

Estas dicotomías, pero especialmente el sueño racionalista, fueron maravillosamente caricaturizadas posteriormente por Voltaire a través de Cacambo, en Cándido. Este representa la sabiduría particular, práctica y popular que se obtiene con la experiencia de la vida y que bien podría equipararse a una posición subjetiva o equivocista y casuística, frente a su opuesto, que es la actitud metafísica del doctor Pangloss, filósofo optimista, pensador teórico, teólogo-cosmo-nigólogo, quien solo habla y teoriza, tanto en situaciones difíciles como frente a las catástrofes.

Cacambo muestra una virtud prudencial basada en la experiencia. Esa prudencia probablemente esté inspirada en el concepto de la sabiduría popular o el buen sentido común que Aristóteles identificara como la frónesis; que es una suerte de sabiduría práctica y prudente que no se opone a la pretensión de las verdades universales, representadas por la sofía, sino que más bien se coloca como su conciencia, como el contrapeso necesario de una reflexión razonable -más que solo racional- y prudente de las pretensiones de universalidad, con base en las particularidades de cada caso. Esta virtud solo puede adquirirse a través de la experiencia práctica cotidiana.

La actitud del doctor Pangloss es una sátira dedicada al optimismo moderno y especialmente a lo que hoy se denomina cientificismo. Es una crítica al pensamiento racionalista ilustrado y una burla al sueño cartesiano de lograr un conocimiento claro y distinto a través de la razón.

Y es que a René Descartes se le recuerda como el padre del racionalismo por el célebre "pienso, luego existo", reducción minimalista de la conclusión que se deriva de la duda radical. La profundidad de esa duda encierra el germen de la negación de los ídolos baconianos y la apertura de una nueva visión del mundo, 
la génesis de un racionalismo extremo que con el tiempo dejó de lado otros saberes, que contribuyó a lo que hoy llamamos modernidad y que tiene como una de sus características esenciales a la ciencia.

Se achaca a Descartes la invención de la visión cientificista del mundo. Es común referirse al cartesio como el representante de la imagen de un mundo máquina cuyo pasado y futuro están determinados como si se tratara de un autómata matemático.

Las críticas de Heidegger a Descartes han contribuido de forma notable a que se observe primordialmente la búsqueda cartesiana hacia un fundamento incuestionable, especialmente clara en sus Meditaciones. Heidegger denomina o pone título a esa actitud moderna del saber como la pretensión matemática, definida como una presuposición fundamental del saber de las cosas (Heidegger 1963, pp. 52-58).

Y efectivamente, con el tiempo esa ha sido una característica esencial de la ciencia moderna. Pero poco ha sido dicho de Descartes en relación a su actitud prudencial y a su interés por el trabajo interdisciplinario, fundamentalmente en lo que se refiere a la necesidad de complementar el empirismo y la reflexión metafísica, lo cotidiano contingente y lo universal inmutable.

La reflexión cartesiana, tanto en las Meditaciones como en el Tratado de la luz, se inicia con la aplicación del principio de análisis, la división de las partes de un problema para enseguida aplicarles la duda radical. Ello consiste en que habrá de desecharse cualquier idea o pretensión de conocimiento apenas se atisbe en ellas la menor duda, no habrá cabida para ningún error que pudiera contaminar los fundamentos del conocimiento, que deberán ser claros, simples y distintos. Este mecanismo permite intuir los elementos primitivos o principios fundamentales, "una concepción de la mente pura y atenta tan fácil y distinta que, sobre aquello que comprendemos, no permanezca la más mínima duda” (AT X, p. 368).

Después, de acuerdo con Descartes, se deberá extraer cuanto se concluya necesariamente de esas intuiciones primarias, pero admitiendo la indeterminación de las leyes particulares porque la validez de los modelos teóricos con que es posible explicar los fenómenos no radica ya en las puras intuiciones sino en su capacidad explicativa, en su verosimilitud, se trata pues de salvar las apariencias fenoménicas. Lo anterior implica un estatuto de certeza problemático y de ninguna manera incontrovertible.

Por una parte, Descartes establece una certeza metafísica de los fundamentos de los fenómenos, pero a la vez admite el carácter hipotético de las leyes particulares. Al abordar los fenómenos desde estas dos posiciones, Descartes echa mano de una exposición sistemática y lógica para postular los principios fundamentales y la persuasión para justificar sus modelos particulares mecanicistas, no sin antes iniciar con la ruptura de los errores de los sentidos, primer nivel de la duda metódica.

Inicia Descartes el Tratado de la luz advirtiendo que puede existir alguna diferencia entre el sentimiento o idea que tenemos de algo y lo que existe en los objetos y produce en nosotros ese sentimiento o idea, se trata de iniciar las con- 
diciones para la duda radical: "Pues, aunque cada cual normalmente se persuada de que las ideas que tenemos en nuestro pensamiento son enteramente semejantes a los objetos de que proceden, no veo ninguna razón que nos asegure que sea así" y añade, introduciendo la duda metódica y apelando a la experiencia: "por contra, observo numerosas experiencias que deben hacernos dudar de ello" (AT XI, p. 4).

Como bien ha notado Peter Dear, Descartes nos provee de diversos ejemplos cotidianos que utiliza como portadores de la evidencia; la experiencia nos sirve como referencia, aunque, paradójicamente, nos vemos obligados a dudar de ella, pues es ella, a la vez, la que nos permite fundamentar la duda. "La experiencia" (Dear 1995, p. 12) se convierte en una base para la contrastación del conocimiento, pero no constituye en sí misma un recurso o fuente de conocimiento.

En ese mismo sentido Daston ha observado que la vida diaria podía proveer de suficientes criterios, aunque imperfectos, de certidumbre moral (Daston 1988, p. xi), es decir, en los que no hubiera duda razonable.

La experiencia tenía también, entre algunos de los virtuosos, incluido Descartes, una importante función reguladora de los juicios a priori. Significaba una prueba o confirmación, tanto en los preceptos de la cristiandad como en la filosofía natural, que no tenía que lograr rigor matemático sino solo estar en el umbral de certidumbre que necesita un hombre razonable en su vida diaria.

Así, Descartes nos ofrece, como ejemplo de la diferencia entre la idea de una cosa y la cosa misma, las palabras. Con las palabras, nos dice, que no tienen ningún parecido con las cosas que significan, concebimos significados sin siquiera darnos cuenta del sonido de sus letras y sílabas; son, a fin de cuentas, signos que producen ideas; de igual forma la naturaleza podría proveernos de signos que nos produzcan el efecto de la luz, sin que esta tenga nada de parecido con lo que la produce.

El interés de Descartes no es demostrar que la luz es diferente a lo que percibimos sino establecer la duda: "no he aportado estos ejemplos para haceros creer absolutamente que la luz sea otra cosa en los objetos que en nuestros ojos, sino solo para que dudéis y, guardándoos de estar preocupados por lo contrario, ahora podáis examinar mejor conmigo lo que es" (AT XI, p. 6).

Descartes añade un ejemplo más, ahora acerca del calor, la sensación que este nos produce es algunas veces de dolor y otras, cuando es moderado, de una especie de cosquilleo, "y no hay nada fuera de nuestro pensamiento que sea semejante a las ideas que concebimos del cosquilleo y del dolor" (AT XI, p. 10).

Descartes ha introducido la duda, nuestra percepción nos engaña, o al menos es posible que así sea, y esa posibilidad es suficiente para dudar radicalmente de ello y darlo por falso. ¿Qué puede salvarse de ser desechado?

No pudiendo ya utilizar la percepción como referencia, puesto que, dada su dudosa veracidad, habrá que desecharla e intuir un principio fundamental que, sin embargo, la explique, Descartes elige el movimiento. Aun la luz y el calor deberán ser explicados con ese principio del movimiento, es decir la sensación de cosquilleo o dolor que nos produce el fuego habrá que atribuirlo al movimiento: "yo, 
que temo equivocarme si supongo alguna cosa más de lo que veo necesariamente que ha de haber, me contento con concebir el movimiento de sus partes" (AT XI, p. 7). Las sensaciones que nos produce, calor deberán explicarse por los distintos modos de movimiento de las partículas de nuestras manos, o de cualquier otro lugar de nuestro cuerpo que puedan producir en nosotros esa impresión.

Siendo la luz y el calor efectos del fuego, deberán ser explicados también con el principio del movimiento de sus partes: "Poned el fuego, poned el calor y haced que arda tanto cuanto queráis: si no suponéis además que alguna de sus partes se mueve y se separa de sus vecinas, no podré imaginar que sufra alguna alteración o cambio" (AT XI, p. 7).

Una vez establecida la duda radical, Descartes afirma como principio fundamental que el movimiento es algo natural que se inició tan pronto el mundo comenzó a existir y es imposible que los movimientos de las partículas que lo forman cesen nunca, sino tan solo que cambien de sujeto transmitiéndoselos unas a otras. Y no es posible concebir que un cuerpo pueda mover a otro si no es moviéndose también a sí mismo. La transmisión del movimiento o conservación de la cantidad de movimiento aparece como una consecuencia de su visión plenista, es decir, concibe el mundo, el espacio, lleno de partículas de diferentes tamaños que llenan en su totalidad cada lugar, cada punto, de tal forma que nada puede moverse si no es desplazando a otros cuerpos. Por supuesto que esta visión plenista no deja de ser problemática, como ha notado Laura Benítez (1993).

Descartes nos obliga a dudar de todo y desecharlo, no asegura nada sino solo los principios fundamentales mínimos que gobiernan el mundo y que Dios habría determinado al principio con la creación. Así, al referirse al vacío tiene el cuidado de no establecer una certidumbre absoluta al decir:

[...] con esto no quiero asegurar que no exista en absoluto el vacío en la naturaleza: solo temo que mi discurso sería demasiado largo si intento explicar este punto y, si bien las experiencias de que he hablado no son tampoco suficientes para probarlo, sí lo son para persuadirnos de que los espacios donde nada sentimos están llenos de la misma materia y contienen -como mínimotanta materia como los que están ocupados por cuerpos que sentimos (AT XI, pp. 20-21).

El cambio, el movimiento, es para Descartes lo fundamental y la razón de que seamos capaces de percibir. Lo que no percibimos o lo que dejamos de percibir es lo habitual, como el calor de nuestro corazón o el peso de nuestro cuerpo. No sentiríamos ningún cuerpo si no fuera a causa del cambio en los órganos de nuestros sentidos (AT XI, p. 21). La percepción es también solo un producto del movimiento, nuestros órganos son golpeados y les es transmitido movimiento que a su vez transmiten. Los cuerpos que nos tocan continuamente pero no producen ningún cambio y por ello no los percibimos.

Más que argumentar la inexistencia del vacío, Descartes nos ofrece con plausibilidad un mundo pleno. Supone la existencia de tres elementos o tipos de corpúsculos con los que es posible el armado del universo. Estos tres elementos son considerados como el mínimo posible, para explicar, junto con el movimiento, 
todos los fenómenos. No es preciso suponer ninguna otra cosa, nos dice Descartes, más que el movimiento, el tamaño, la figura y la disposición de sus partes. $\mathrm{Si}$, como es posible imaginar, hay una gran cantidad de diminutos intervalos entre las partes de que está compuesto un cuerpo sólido, la experiencia nos indica que estos están llenos de aire y, si entre las partes del aire hay también una gran cantidad de intervalos entre sus partes, estos no pueden estar vacíos por lo que es posible concluir que hay necesariamente otros cuerpos mezclados con el aire, los cuales llenan tan completamente como es posible los diminutos espacios que hay entre sus partes (AT XI, p. 23).

Todo esto es parte de la retórica cartesiana que busca persuadir al lector de la capacidad explicativa del modelo: "Tampoco es preciso pensar que los elementos carezcan de lugar en el mundo que les esté particularmente destinado y donde puedan conservarse en su pureza natural” (AT XI, p. 28).

Así como en los primeros momentos de la "Primera Meditación" Descartes supone la existencia no de Dios, "fuente suprema de la verdad", sino de un "cierto genio maligno", en la primera parte de El mundo en el Tratado de la luz supone la existencia del genio maligno, aunque sin mencionarlo, y nos propone, para alejarnos de él y sus engaños, alejarnos de los sentidos y los prejuicios trasladándonos hacia un mundo ficticio. La tesis cartesiana es que en ese mundo fábula tendremos la posibilidad de saber qué es lo verdadero. Se trata de una medida radical persuasiva que permita hacer más verosímiles sus opiniones. Así, Descartes nos propone una especie de salto ontológico al olvidarnos del mundo real y pensar en una fábula, en otro mundo en el que sea posible desprendernos de todo el conocimiento que suponemos verdadero de este mundo. Es otro universo que Descartes irá describiendo a partir de los elementos fundamentales que le componen y sus leyes particulares acordes con los principios generales de la conservación de materia en sus tres formas o elementos corpusculares y el movimiento, y así va mostrando que en ese mundo de fábula los fenómenos son idénticos a los de este mundo real y, por tanto, las explicaciones que nos otorga acerca de su funcionamiento bien pueden ser las de este mundo.

Su objetivo no es el de explicar las cosas que existen efectivamente en el verdadero mundo, nos dice Descartes, sino solo fingir uno en el que nada haya que los espíritus más comunes no sean capaces de concebir y que pueda, no obstante, ser creado tal como es imaginado o fingido. Pero en ese mundo sería imposible poner la menor cosa oscura (inexplicable), porque podría ocurrir que, mediante esa oscuridad, hubiera alguna contradicción escondida de la no se percatase y, de este modo, podría suponerse alguna cosa imposible; en cambio, al poder imaginar todo lo que se pone en ese mundo, aun cuando no tenga nada de común con el verdadero, Dios puede crearlo en uno nuevo puesto que puede crear todas las cosas que podemos imaginar (AT XI, p. 36).

Descartes se aleja del sentido común y los prejuicios e inventa un mundo que, si bien pudiera parecer totalmente distinto al nuestro, poco a poco se observa que las leyes que obedece (por lejanas o absurdas que parezcan) dan lugar a este mundo nuestro. 
Descartes utiliza simultáneamente los principios metafísicos y la confirmación empírica de su verdad a través de ejemplos prácticos persuasivos. Sin embargo, el estatuto de certeza de los mecanismos particulares de los fenómenos, más allá del cumplimiento de los principios generales, es incierto. En todo caso se trata de una certeza moral cuya diferencia con la certeza metafísica Descartes establece con claridad:

[...] sin embargo, a fin de no cometer ninguna injusticia a la verdad suponiéndola menos cierta de lo que es, distinguiré aquí dos tipos de certeza. La primera es la llamada moral, esto es, suficiente para regular nuestras costumbres: es tan extensa como las cosas de las que no tenemos costumbre de dudar acerca de la conducta de la vida, aunque sepamos que podría ocurrir, hablando absolutamente, que fueran falsas.

El otro tipo de certeza se da cuando pensamos que es totalmente imposible que la cosa sea distinta de cómo la juzgamos. Está fundada sobre un principio metafísico bien asegurado: Dios, siendo soberanamente bueno y fuente de toda verdad, dado que nos ha creado; es cierto que la potencia o facultad que nos ha dado para distinguir lo verdadero de los falso no se equivoca cuando la usamos correctamente y nos muestra con evidencia que una cosa es verdadera (AT IX-2, pp. 323-324).

En sus Principios, Descartes deja en claro esa actitud prudencial; nos dice que cuanto escribe son hipótesis que bien podrían estar alejadas de la verdad pero, aunque así fuera, si las cosas deducidas de tales hipótesis concordaran enteramente con las experiencias, no serían menos útiles que si fueran verdaderas para explicar y predecir los fenómenos (AT IX-2, p. 123).

Las reglas mediante las que Dios hace actuar a la naturaleza en el nuevo mundo cartesiano son necesarias y bastan para hacernos conocer las restantes; su estatuto de certeza no está en duda: la primera regla establece que cada parte de materia permanece siempre en un mismo estado mientras el encuentro con otras no le obligue a cambiarlo, en este caso, si una parte de materia se encuentra en movimiento, continuará en ese estado de movimiento hasta que otra parte de materia lo modifique. El movimiento no se destruye, solo se transmite entre los pedazos de materia. La segunda regla complementa la primera: cuando un cuerpo impele a otro no puede darle ningún movimiento si él no pierde simultáneamente igual cantidad del suyo.

Aunque todo lo que nuestros sentidos han experimentado en el mundo verdadero (de los sentidos y apariencias), dice Descartes, parecería ser manifiestamente contrario a las dos reglas anteriores, la razón nos las muestra tan fuertes que es imposible no suponerlas en ese nuevo mundo.

La tercera regla establece que, cuando un cuerpo se mueva siguiendo una curva, cada una de sus partes tiende a proseguir su movimiento en línea recta. Y enseguida nos persuade con ejemplos de una rueda girando y de una honda. De todos los movimientos solo el rectilíneo es enteramente simple.

Las contribuciones cartesianas indudables a la noción moderna del mundo y especialmente de la física son, en primer lugar, el reconocimiento de la necesi- 
dad de separar el problema en partes, admitiendo que la solución a un problema complejo se reduce al tratamiento de sus partes por separado; en segundo lugar, la visión mecanicista en la que es posible explicar los fenómenos naturales atendiendo exclusivamente a su estructura fundamental corpuscular como agregado material de partículas, y a su movimiento geometrizable.

Sin embargo, el salto ontológico cartesiano a través de la duda radical constituye un elemento fundamental en el rompimiento con el modelo medieval del conocimiento; se trata de una aportación revolucionaria que permite el cuestionamiento de los paradigmas normales, parafraseando a Kuhn. Y como última reflexión habrá que valorar la humildad cartesiana al reconocer que sus explicaciones de los particulares no son sino hipótesis heurísticas para comprender el mundo, cuyo estatuto de certeza es solo moral y, por tanto, no se trata de verdades incontrovertibles y absolutas. Sin duda que lo anterior enaltece la obra cartesiana y desmitifica el dogmatismo que la crítica a la modernidad le ha achacado.

\section{Bibliografía}

Benítez, L. (1993), El Mundo en René Descartes, México: IIF-UNAM.

Boyle, R. (1991), Selected Philosophical Papers of Robert Boyle (ed. M. A. Stewart), New York: Barnes and Noble.

Boyle, R. (1996), A Free Enquiry into the Vulgarly Received Notion of Nature (ed. B. D. Edward y M. Hunter), Cambridge: Cambridge University Press.

Daston, L. (1988), Classical Probability in the Enlightenment, Princeton: Princeton University Press.

Dear, P. (1995), Discipline and Experience, Chicago: The University of Chicago Press.

Descartes, R. (1989), El mundo. Tratado de la luz, Barcelona: Anthropos.

Heidegger, M. (1963), La pregunta por la cosa, Buenos Aires: Memphis.

Hooykaas, R. (1949), "The Experimental Origin of The Chemical Atomic and Molecular Theory Before Boyle", Chymia 2: 65-80.

Jara, G.S. (2009), La ciencia prudencial de Robert Boyle, Morelia: Universidad Michoacana de San Nicolás de Hidalgo.

Sargent, R. M. (1995), The Diffident Naturalist, Chicago: The University of Chicago Press.

Shapiro, B. J. (1991), Beyond Reasonable Doubt and Probable Cause. Historical Perspectives on the Anglo-American Law of Evidence, Berkeley: University of California Press.

Turró, S. (1989), "Estudio Introductorio”, en Descartes (1989), pp. 7-42. 